\title{
Protein: a versatile biopolymer for the fabrication of smart materials for drug delivery
}

\author{
BEENA G SINGH ${ }^{\mathrm{a}, *}$, RAM P DAS ${ }^{\mathrm{a}, \mathrm{b}}$ and AMIT KUNWAR ${ }^{\mathrm{a}, \mathrm{b}}$ \\ ${ }^{a}$ Radiation and Photochemistry Division, Bhabha Atomic Research Centre, \\ Trombay, Mumbai 400 085, Maharashtra, India \\ ${ }^{\mathrm{b}}$ Homi Bhabha National Institute, Anushaktinagar, Mumbai 400 094, Maharashtra, India \\ E-mail: beenam@barc.gov.in
}

MS received 30 March 2019; revised 6 June 2019; accepted 13 June 2019

\begin{abstract}
Drug delivery is a broad field which deals with the delivery of pharmaceutical compounds to the desired site with an aim to achieve maximum therapeutic effect and minimum toxicity in treating a diseased condition. To achieve the goal, a carrier system is required for controlled and sustained drug release. Polymers are an all-time favourite matrix used to control the release of drug at the desired rate. Among the polymers, the biopolymers derived from proteins are attracting considerable attention due to their utility in delivering vaccine and chemotherapeutics in clinical application. Additionally, they are biocompatible, biodegradable, and in certain cases, cost-effective. The review addresses the source, structure and characterization of different proteins used as drug delivery carriers. Along with this, the factors contributing to the release of drug from the protein carrier has also been discussed.
\end{abstract}

Keywords. Protein; silk; gelatin; albumin; keratin; zein; casein; porosity; molecular weight; particle size.

\section{Introduction}

Drugs are chemical compounds used to treat, control or prevent disease conditions. To increase their therapeutic efficacy, scientists are designing new drugs. However, the procedure of drug discovery is a cumbersome process. Even after recent advances in designing potent drugs with the aid of bioinformatics and novel chemical reactions, many compounds fail at the preclinical evaluation due to low solubility, poor stability, short circulation time, and non-specific toxicity. Another way to improve the efficacy of existing drugs is being explored by formulating the drug with a suitable excipient that can deliver a drug at a controlled rate to the specific site. ${ }^{1,2}$ This process of delivering the therapeutics with an aim to increase its efficacy and potency is called as drug delivery. In drug delivery, the carriers are selected in such a way that the therapeutics are released at the desired site with an optimum rate and have minimum toxicity. ${ }^{1,2}$ The main challenge in developing a suitable drug carrier is to design them to improve the pharmacokinetics and pharmacodynamic properties of the therapeutics. In this regard, polymers in reservoir-based drug delivery

*For correspondence are the main choices due to their complex structures which can load and release both hydrophobic as well as hydrophilic therapeutics improving the pharmacokinetic properties. They are known to have improved circulation time than the conventional small drug molecules thus facilitates better drug availability. Tremendous use of polymers has been witnessed in the area of polymer therapeutics and nanomedicines. ${ }^{3}$ Among the polymers, the general characteristic feature that makes them a potential candidate for drug delivery include safety, efficacy, hydrophilicity, absence of immunogenicity, suitable pharmacokinetics, and the presence of functional groups for covalent conjugation of drugs, targeting moieties, or formation of copolymer. The mode of application of these drugs may vary depending upon the disease origin and condition. On basis of this, the drug administration may be parenteral, topical or enternal, which subsequently demands the drug to be encased either in solution phase or in the form of ointment or gel.

Protein particles offer multiple advantages over other biopolymers and synthetic polymers such as high loading capacity, ease of production and non-immunogenic. Additionally, proteins are good raw materials since they have the advantages of 
absorbability and low toxicity of amino acid byproducts on degradation by protease. Through a variety of aqueous or organic solvent processing methods, natural protein composites can be fabricated into the formats of hydrogels, tubes, sponges, composites, fibres, microspheres and thin films which can be used for a wide range of applications. For example, gelatinbased materials are reported to be fabricated in the wide range of form from nanoparticles, microparticles, macroparticles, film, hydrogels, hard and soft capsules, lozenge, etc. Protein-based materials can be easily manipulated and there are various possibilities for surface alteration and/or modification for covalent drug attachment. ${ }^{4-8}$

This review aims to provide an overview of different proteins that are used for drug delivery, critically reviewing the recent developments in factors affecting the drug releasing capacity of bio-polymers.

\section{Classification of protein}

Proteins used for fabricating materials can be classified as animal and plant proteins (Scheme 1). In the case of animal proteins, the low toxicity of the end product (metabolite or product of degradation), and easy manipulation to load both hydrophobic and hydrophilic drug gives them an edge over synthetic polymers. ${ }^{8}$ The major drawback of animal proteins is the risk of infection from pathogen contamination. However, in some cases, it is not actually a real problem since animal proteins can be sterilized. On the other hand, proteins derived from plants are more hydrophobic in nature and can be used to deliver water-insoluble drugs or nutrients. Due to hydrophobic nature, plant proteins can be fabricated into desired materials like nanoparticles or hydrogel without using toxic chemical cross-linking agent.

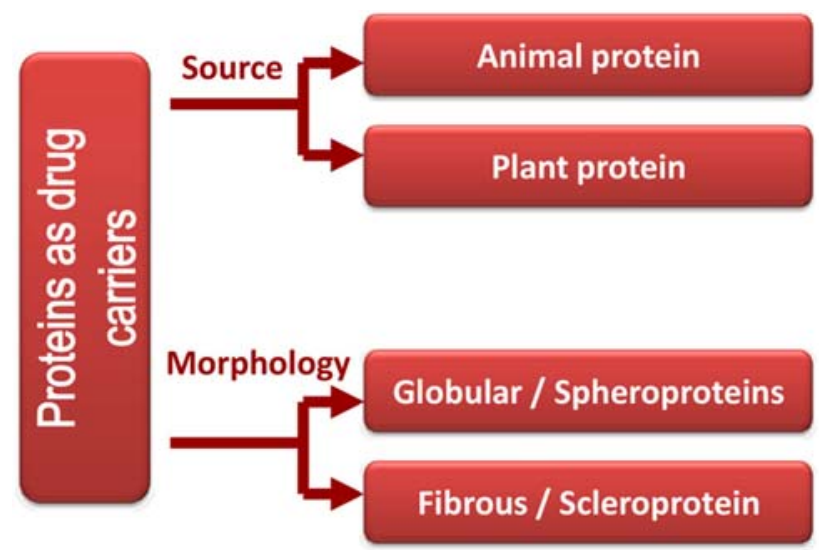

Scheme 1. Classification of proteins for drug delivery.
In addition, plant proteins are less expensive than animal proteins. $^{8}$

Another way to classify protein can be based on their structure or morphology (Scheme 1). ${ }^{9}$ The two types of proteins used for drug delivery are globular and fibrous proteins. Globular proteins or spheroproteins are spherical proteins and possess some water-solubility or form a colloidal suspension in water. These proteins are generally functional in nature. Certain enzymes, transporters and carrier proteins belong to this group. They are mostly used to prepare nanoparticles and microparticles. The most extensively studied globular proteins used for drug delivery are albumins. Fibrous or scleroprotein functions mainly as structural proteins or storage proteins. They are long protein filaments, where the quaternary structure looks like rods or wires. They are typically inert and water-insoluble. The peptide sequence in a scleroprotein generally has limited residues with repeats and can form unusual secondary structures, such as a triple helix in collagen. The structures often feature cross-links between chains (disulfide bonds between keratin chains). Scleroproteins tend not to denature as easily as globular proteins. They can be easily fabricated as films or hydrogels. Some methods are reported in the literature to prepare nano- and microparticles. Proteins such as keratin, collagen, elastin, etc., are examples of scleroprotein.

\section{Different proteins used in drug delivery}

\subsection{Gelatin}

Gelatin is a derived and partially denatured protein formed by the hydrolysis of structural protein collagen. The amino acid composition of gelatin is similar to collagen. However, the source (i.e., porcine, bovine or fish) and the manufacturing process affects the nature and quality of the gelatin. The breakdown of collagen to gelatin can be carried out by acid or alkaline hydrolysis, forming two different forms namely, gelatin $\mathrm{A}$ and gelatin $\mathrm{B}$, respectively. ${ }^{10,11}$ Extensive enzymatic degradation of gelatin results in gelatin hydrolysates. The average molecular weight of gelatin A and B lies in the range $19 \mathrm{kDa}$ to $100 \mathrm{kDa}$, while that of gelatin hydrolysates is less than $5 \mathrm{kDa}$. Gelatin consists of 19 amino acids predominated by glycine (Gly), proline (Pro) and hydroxyproline (Hyp), which consists of around $50 \%$ of amino acid content. Along with these, it contains 8 essential amino acids including lysine and arginine (Arg). It lacks tryptophan and is deficient in isoleucine, threonine and methionine. Due to non-specific breakdown of collagen, gelatin, in general, is a heterogeneous mixture of single or multi-stranded polypeptide having repetitive amino acid sequence of -Ala-Gly-Pro-Arg-Gly-Glu- 
Hyp-Gly-Pro-, where glycine is found at every third position. The presence of -(Gly-Pro-X)- and -(Gly-XHyp-) sequence is responsible for the formation of right-handed triple helix structure. The presence of Gly is required to allow the three chains to come close, while the ring structure in Pro and Hyp gives enhanced rigidity to the structure. The exact amino acid composition varies greatly, depending on the species of origin. Alkaline pre-treatment converts asparagines and glutamine residues to their respective acids and results in a higher viscosity of gelatin B as compared to gelatin A. Mechanical properties of gelatin gels are quantified using the bloom test and are sensitive to temperature. ${ }^{10-12}$ Gelatin type $\mathrm{A}$ has an isoelectric point (IEP) of $\sim 8.0$ and gelatin type $\mathrm{B}$ has an IEP of $\sim 4.9$. The secondary structure of gelatin can be studied by circular dichroism (CD). Gelatin solution exhibits a negative peak at $205 \mathrm{~nm}$ and a positive peak at $222 \mathrm{~nm}$ which corresponds to the presence of random coil and triple helix state, respectively. During the fabrication of gelatin into different materials, CD can be used to explore the changes in the secondary structure. For example, Sakai et al., reported that formation of gelatin microgel from neat gelatin solution resulted in the decrease of the helix structure to random coil state. ${ }^{13}$ Finally, gelatin chains contain motifs such as Arg-Gly-Asp (RGD) sequences that help to adhere to cells, thereby improving the interaction between gelatin and cell membrane. ${ }^{10}$

\subsection{Albumin}

Albumin is a carrier protein which can be obtained from a variety of sources including egg white (ovalbumin), bovine serum albumin, and human serum albumin (HSA). Among them, the extensively studied, HSA is the most abundant protein in human blood (30-50 g/l serum) and the function of this protein is to deliver solutes from the bloodstream to their target organs, as well as to maintain the $\mathrm{pH}$ and osmotic pressure of plasma. It is a non-glycosylated singlestranded peptide containing 565 amino acids that fold to form a heart shape protein $(\sim 66.5 \mathrm{KDa})$. HSA has one cysteine and tryptophan amino acid along with 17 disulfide bond. It has a high content of polar amino acid-like aspartic acid (Asp) and glutamic acid (Glu), histidine (His), lysine (Lys) and arginine (Arg) which is responsible for its high solubility at $\mathrm{pH} 7$ (up to $40 \% \mathrm{w} / \mathrm{v})$. The high content of Aspartic and glutamic acid is responsible for the overall negative charge on HSA at neutral $\mathrm{pH}$. Further, $\sim 70 \%$ of the tertiary structure in HSA is helical due to high content of leucine and lysine. The presence of 17 disulfide linkages is responsible for its higher stability and can be heated up to $60{ }^{\circ} \mathrm{C}$ for $10 \mathrm{~h}$ without undergoing thermal denaturation. ${ }^{14}$ The IEP of HSA is 4.7. The melting point of $\mathrm{HSA}$ is $65{ }^{\circ} \mathrm{C}$, above which it undergoes irreversible denaturation. The half-life of HSA in human blood is around 19 days. This long half-life is thought mainly due to neonatal Fc receptor (FcRn)-mediated recycling, and the Megalin/Cubilincomplex present in the proximal tubule of the kidney. They are gaining importance in anticancer drug delivery due to their ability to accumulate selectively in tumour sites, via complementary pathways such as enhanced permeation retention (EPR) effect, and receptor (gp60 and SPARC) mediated transcytosis. ${ }^{15,16}$ Albumin contains multiple hydrophobic binding pockets and naturally serves as a transporter of variety of different ligands such as fatty acids and steroids as well as different drugs. In addition to its ordinary clinical applications, such as hypovolemic shock treatment, many investigators have attempted to utilize HSA as a carrier to deliver various drugs to their specific targets. Some of the successful albumin nanoformulations in clinic are Abraxane (albuminbound formulation of paclitaxel) and Aldoxorubicin (albumin-bound to doxorubicin). ${ }^{16}$ Albumin is widely used in the preparation of nanospheres and nanocapsules. These albumin nanocarriers are non-toxic, biodegradable, easy to prepare of well defined sizes, nonimmunogenic, and also carry reactive group (thiol, amines and carboxyl) which can be utilized to bind specific receptor or to modify surface properties. The drug entrapped in the albumin nanoparticle can be easily digested by the enzyme protease. The albuminbased nanocarriers can help to increase the aqueous solubility of hydrophobic drugs, control sustained release and improve pharmacokinetics parameters. ${ }^{17,18}$

\subsection{Keratin}

Keratin-based materials are investigated for drug delivery due to their intrinsic biocompatibility, biodegradability, mechanical durability and natural abundance. It is a structural protein found in hair, nails, horns, claws, hooves and the outer layer of human skin. Keratin is a fibrous protein and can be classified as $\alpha$-keratin (occurs in mammals) or $\beta$ keratin (occurs in birds and reptiles). Of these keratins, the $\alpha$-keratin is well-studied and the wool keratins are extensively investigated for drug delivery. Further $\alpha$-keratins are further classified as Type I (acidic) and Type II (basic/neutral). Irrespective of 
the type, $\alpha$-keratin polypeptides are rich in alanine, leucine, arginine, cysteine and form the right-handed helix. They also have high contents of glycine, proline and serine, but have low content of lysine, histidine and methionine, while tryptophan is barely present. Two of these polypeptides twist themselves to form left-handed helical structure or heterodimers, which are also referred as the coiled-coil structure. These helix keratins have inter-molecular bonding of the disulfide cysteine amino acid and inter- and intramolecular bonding of polar and non-polar acids. Cystine has an important role in determining the physicochemical properties of keratin. Wool proteins are generally inert on exposure to chemical and physical factors. ${ }^{19}$ These proteins are insoluble in water and in many weak acids, alkali solutions or organic solvents, as well as resistant to common protein-digesting enzymes such as pepsin or trypsin and intra-chain cross-links of cysteine disulfide bonds. In wool keratin, different interactions occur between the amino acid such as hydrogen, hydrophobic and ionic bonds, which plays an important role in the stability and properties of the wool keratin. The existence of ionic bonds is $\mathrm{pH}$ dependent and is observed maximum at the IEP of 4.9 when the protein is in the form of zwitterions $\left({ }^{+} \mathrm{H}_{3} \mathrm{~N}-\mathrm{CHR}-\mathrm{COO}^{-}\right)$, while under extreme acidic or basic conditions, the ionic bonds are at their lowest level. The ionic bond occurs between carboxylate and protonated amino group, which can be reduced at extreme acidic and alkaline $\mathrm{pH}^{20}$ The disulfide bonds give keratin a compact three-dimensional structure and provide high resistance to chemical or enzymatic reactions. Wool keratin with $4-8$ wt\% sulfur is known as hard keratin whereas keratin found in the epidermal tissue of skin has $2 \%$ sulfur and $50-75 \%$ moisture and is considered as soft keratin. Wool solubilisation occurs by disruption of the complex keratin structure by reduction, oxidation, microwave irradiation, alkali extraction, steam explosion, sulfitolysis and ionic liquids. Keratin obtained from the wool fibre can be classified into four different molecular weight groups: a low sulfur content (LS) with a MW of $45-60 \mathrm{kDa}$, a fraction with a high sulfur content with a MW of 11-28 kDa, and fractions with a high glycine or high tyrosine content with a MW of 9-12 $\mathrm{kDa}$. The presence of cell adhesion sequences such as arginine-glycine-aspartic acid (RGD) and leucine-aspartic acid-valine (LDV) in the natural sequence of keratin also add to its potential to be used as a matrix for cell growth and thus keratin-based materials can be used for the development of tissue engineering constructs. $^{21}$
Keratin structure is hydroscopic in nature and it exhibits glass transition temperature that is dependent on the water content (water acts as a plasticizer). Dry wool has a glass transition temperature of about $170{ }^{\circ} \mathrm{C}$, while when wet it is about $-10{ }^{\circ} \mathrm{C}$. ${ }^{22}$

\subsection{Casein}

Milk proteins are preferably used in food industries due to their ability to bind bioactive nutrients. Among the known milk proteins, casein - a phosphoprotein, is the most preferred as it is inexpensive, readily available, non-toxic, highly stable and easily digestible. In general, bovine milk contains $3.2 \%$ of protein, out of which casein proteins constitutes around $80 \%$ of the total protein. The remaining is whey protein which constitutes of $\alpha$ lactalbumin, $\beta$-lactoglobulin, serum albumin and immunoglobulins. In bovine milk, casein comprises of four types of protein known as $\alpha_{\mathrm{S} 1}, \alpha_{\mathrm{S} 2}, \beta$ and $\kappa$ casein which differ in their amino acid, phosphorous and carbohydrate content. Their molecular weights are in the range of 19 to $25 \mathrm{kDa}$ with average IEP in the range of 4.6 and 4.8. At neutral $\mathrm{pH}$ or in milk $(\mathrm{pH}$ 6.6) the caseins are negatively charged. These proteins collectively exist in milk in an aggregated form known as casein micelles. These proteins are bonded by hydrophobic interactions and colloidal calcium phosphate (CCP) that are attached to the phosphorylated serine residues of the casein side chains. CCP plays a crucial role in maintaining micellar integrity. Three of the caseins $(\alpha \mathrm{S} 1-, \alpha \mathrm{S} 2-$ and $\beta$-casein) contain centers of phosphorylation (at least three phosphoserine residues in close proximity) that can bind to the amorphous calcium phosphate cluster. Both $\alpha_{\mathrm{S}^{-}}$and $\alpha_{\mathrm{S} 2}$-casein contain more than one phosphate center and can thus act as linking agents between nanoclusters. The surface of the micelles is primarily covered with $\kappa$-casein providing a hydrophilic, charged surface layer which stabilizes the micelles through inter-micellar electrostatic and steric repulsion. Casein proteins have distinct hydrophobic and hydrophilic domains. $\alpha_{\mathrm{S}^{-}}$ casein has a strongly acidic peptide of 40 amino acids that contains seven of the eight phosphate groups, twelve carboxyl groups and only four positive groups. The highly charged $\mathrm{N}$-terminal region of $\beta$-casein contains four of the five phosphates of the molecule, seven carboxyl groups and only two positive groups. The sialylated glycoprotein $\kappa$-casein has only one phosphate and fourteen carboxylic acid groups located in the hydrophilic C-terminal region 
called the glycomacropeptide. ${ }^{23}$ Due to the selfassembled structure, caseins are amphiphilic in nature. They are the part of milk transport proteins through which new born babies obtain important minerals like calcium and phosphate at higher concentration. Caseins are rich in proline and sulfurcontaining amino acids are absent which results in undefined secondary and tertiary structure. The high concentration of proline makes the $\alpha$-helical and $\beta$ sheets structure non-viable making casein flexible and present in random coil form. ${ }^{24}$ These features attribute to the presence of several energetically favourable conformations in solution, which are responsible for the high-temperature stability of casein and it is also classified as rheomorphic proteins. Additionally, the presence of high content of proline results in its easy degradation by protease, which along with the acid-soluble calcium-phosphate bridging, makes an excellent target-activated release mechanism for unloading the drug in the stomach. With all the advantages of using casein as a drug delivery agent, the possibility of immunogenicity/allergenicity response limits its commercial success as a drug delivery agent. ${ }^{25}$

\subsection{Silk protein}

Silk is fibrous protein secreted by different types of insects and spider. The proteins in raw silk derived from silkworm (Bombyx mori) are made up of supramolecular assembly of quaternary structures derived from two different types of proteins, fibroin and sericin. ${ }^{26,27}$

Silk fibroin is made up of a heavy polypeptide chain $(\sim 325 \mathrm{kDa})$ and a light polypeptide chain $(25 \mathrm{kDa})$ joined by a single disulfide bond at the $\mathrm{C}$-terminus of the heavy chain polypeptide. A glycoprotein p25 $(25 \mathrm{kDa})$ is non-covalently attached to the heterodimer complex. The primary peptide conformation is mainly due to eighteen amino acids. The heavy polypeptide chain is hydrophobic in nature due to the repetitive sequence of Gly-Ala-Gly-Ala-Gly-Ser and repeats of Gly-Ala/Ser/Tyr dipeptides, which makes up $90 \%$ of the silk. They form stable $\beta$-sheet plated core, while the light polypeptide chain rich in amino acid-like glutamic acid, valine, and aspartic acid has random coil-like structure. The light polypeptide chain is more hydrophilic in nature and flexible compared to heavy polypeptide chain. These amino acids are used as side chains and affect properties such as elasticity and strength. The $\beta$-sheet structure is responsible for the mechanical strength of the silk fibre. The IEP of fibroin protein had been demonstrated to be 4.53 and overall negative charge surface at neutral $\mathrm{pH}^{26,28}$

Silk sericin is a globular protein and is easily soluble in water. The molecular weight of sericin is greater than $200 \mathrm{kDa}$ and consists of 18 amino acid similar to fibroin but differs in its composition. Unlike fibroin, the amino acids in sericin are more polar (78\%) and mainly consists of serine and aspartic acid. The composition of the amino acid also depends on the silkworm variety. Sericin occurs mainly in an amorphous random coil and to a lesser extent in a $\beta$-sheet structure. The randomly coiled structure easily converts to $\beta$-sheet structure on exposure to moisture and mechanical stretching. Random coil structure is stable at a higher temperature and on decreasing the temperature converts to $\beta$-sheets, resulting in gel formation. Due to this, it exhibits temperature dependant sol-gel property. However, the transition temperature of this is dictated by the extraction process, concentration and molecular weight of the extracted sericin. As the acidic residues are dominant in sericin, the IEP of the protein is reported to be $\mathrm{pH} 4$ and its surface has negative charge at neutral $\mathrm{pH} .{ }^{27,28}$

\subsection{Zein}

Zein is an important storage protein from maize and comprises of $50 \%$ of corn protein. Corn consist of four proteins, Zein (present in endosperm of corn seed), Gliadin (distributed between endosperm and germ layer), Albumin and Glogumin (present in germ). It is a US-FDA approved biomaterial having a diverse industrial utility such as plastics, paper coatings, adhesives, substitutes for shellac, laminating board, solid colour printing films and food industry. Zein is a prolamine protein and is deficient in essential amino acid like lysine and tryptophan and this makes it poor in nutritional quality. It is an alcohol soluble protein and practically insoluble in water. However, its solubility in water can be enhanced in the presence of alcohol, high concentration of urea or alkali ( $\mathrm{pH} 11)$ or in the presence of anionic detergents. The hydrophobicity of zein is due to its amino acid composition. It is rich in non-polar amino acids such as glutamic acid $(21-26 \%)$, leucine $(20 \%)$, proline $(10 \%)$ and alanine $(10 \%)$. It is deficient in acidic and basic amino acid. ${ }^{29}$

Zein is a heterogeneous aggregate of different peptides linked by disulfide bonds with a molecular weight of about $40 \mathrm{kDa}$. During the processing of native zein from corn, different protein fractions have been found. These fractions differ in solubility, 
molecular weight, immunological response and structure. Depending upon the differential solubility in aqueous propyl alcohol with different $\mathrm{pH}$ values, urea and salt concentration, the protein fractions are classified into $\alpha$-zein, $\beta$-zein, $\gamma$-zein and $\delta$-zein. Among these the $\alpha$ - and the $\beta$-zein are well-characterized and extensively studied, while limited information is available on the $\delta$-zein. The $\alpha$-zein constitutes of $75-85 \%$ of the total zein. It is soluble in $40-50 \%$ isopropyl alcohol and has molecular weight of $20-24 \mathrm{kDa}$. The IEP of this fraction is at $\mathrm{pH} 6.8$. The secondary structure of the $\alpha$-zein exists mainly in $\alpha$ helix structure $(57 \%)$, followed by $\beta$-sheets and random coil which constitute for 7.1 and $8.2 \%$. Approximately $28 \%$ of the secondary structure could not be determined. $\beta$-zein is soluble in $30-80 \%$ isopropyl alcohol under reducing condition. It represents $1-15 \%$ of the total zein and constitutes of methionine rich polypeptides with molecular weight of 17-18 kDa. $\gamma-$ zein is soluble in $0-20 \%$ isopropyl alcohol under reducing condition. It constitutes $5-10 \%$ of the total zein and is a proline-rich fraction with a molecular weight of $27 \mathrm{kDa}$. The N-terminal of the $\gamma$-zein is found to interact with the cell membranes and has served as peptide carrier for drug across cell membrane. The $\delta$-zein is around $10 \mathrm{kDa}$ and rich in methionine residue. ${ }^{30}$

\section{Method of preparation of protein nanoparticles}

There are several different techniques that can be used to prepare protein nanoparticles such as desolvation or coacervation, emulsification, self-assembly, electrospraying, salt precipitation, etc. (Scheme 2). ${ }^{31}$ Some of the most commonly used methods are briefly explained below. In the desolvation process, desolvating agents like acetone or alcohol are used to dehydrate the protein which results in its aggregation. The aggregates are then cross-linked using chemical cross-linkers like glutaraldehyde, polyphenols, etc. Although it is the most commonly used method, the use of organic solvent and toxic crosslinking agents like aldehyde are the main limitation of the method. Similar to desolvation process, in the coacervation process, the protein is aggregated by exploring the difference in protein solubility in a solvent. The difference can be achieved by changing the solvent polarity, $\mathrm{pH}$, ionic strength or presence of electrolytes. Proteins nanoparticles from albumin, gelatin, silk, whey, gliadin and casein are prepared by this method. Salt precipitation is one of the simplest methods to prepare protein nanoparticles. Similar to desolvation or coacervation method, in this method the hydrophilicity of the protein is reduced by adding salt which neutralizes the charge on the protein and

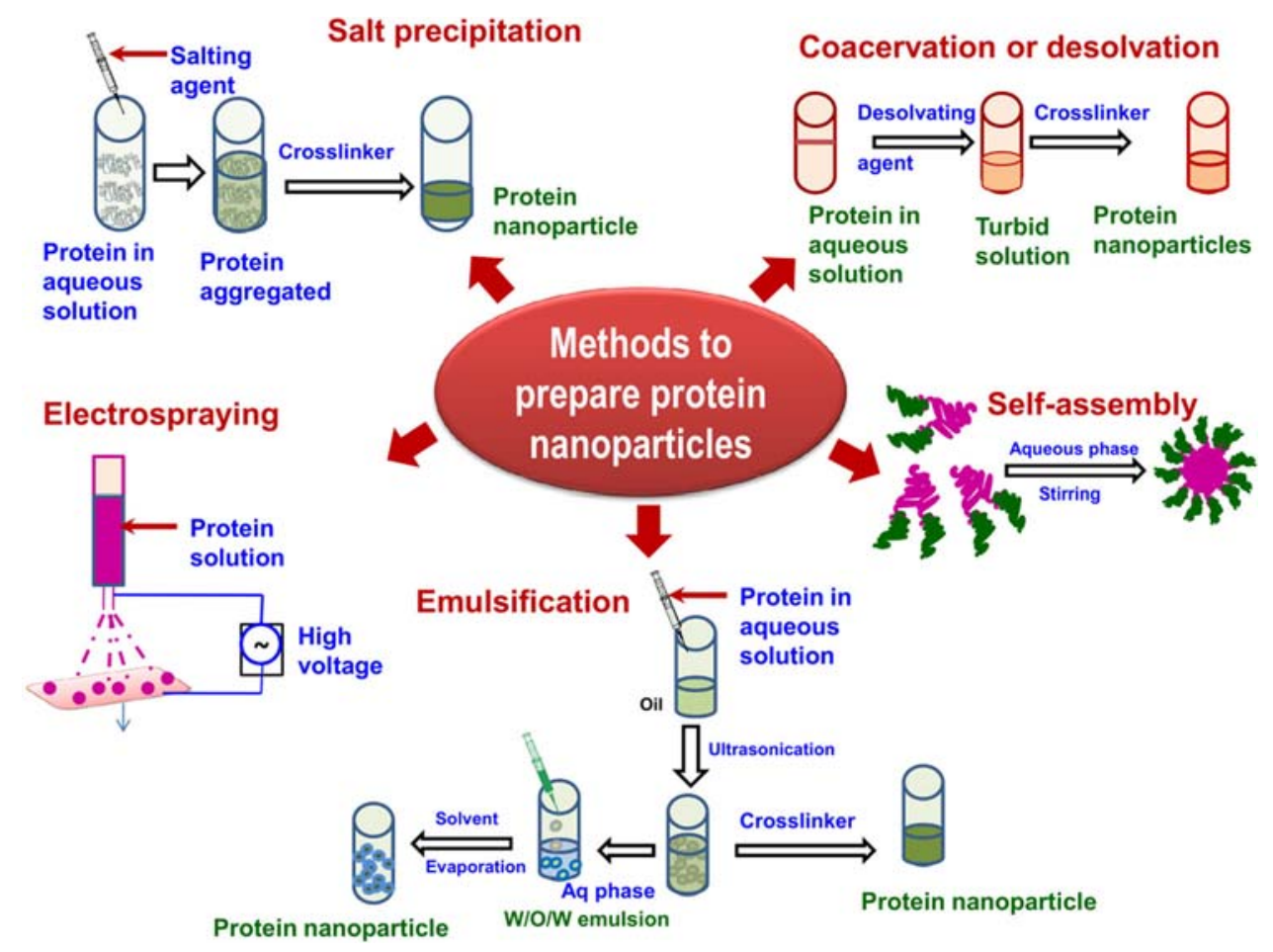

Scheme 2. Different methods to prepare protein nanoparticles. 
induces hydrophobic interaction between two protein molecules. In this method, care has to be taken to check whether the addition of the salting agent changes the bioactivity or conformational structure of the protein or not. $^{32}$

In the emulsification process, the protein dissolved in an aqueous solution is homogenized in an oil phase or organic solvent by using a high-pressure homogenizer or ultrasonication to form water in oil emulsion (W/O). The nanoparticles formed at the interface can be stabilized by chemical crosslinking or thermal denaturation of the protein. Additionally, emulsifiers like Span 80, phosphatidylcholine are used to stabilize the emulsion. The protein nanoparticles formed in the $\mathrm{O} / \mathrm{W}$ emulsion can be further homogenized in an aqueous phase to form the double emulsion $(\mathrm{W} / \mathrm{O} / \mathrm{W})$. The size of the protein particles formed by this method is bigger as compared to that formed by desolvation or coacervation method. The nanoparticles derived from albumin, gelatin, collagen, etc., are known to be prepared by this method. ${ }^{8}$

Self-assembly is the most common method used for the preparation of casein, zein and keratin nanoparticles. As caseins are negatively charged at neutral $\mathrm{pH}$, they bind with high concentrations of calcium phosphate, via self-assembly to form micelles. Proteins below and above their IEP are positively and negatively charged, respectively. Oppositely charged proteins can selfassemble via electrostatic attraction to form nano-suspension. Also, hydrophilic proteins like gelatin, albumin on conjugation with polyethylene glycol are modified to obtain amphiphilicity. Such modified protein undergoes self-assembly to form micellar shaped nanospheres when added to an aqueous solution. ${ }^{33}$

Electro-spray drying is one of the well-established scalable methods used in the pharmaceutical industry for producing a dry powder from a liquid phase. The process utilizes atomization of a liquid jet into fine droplets under the influence of the high voltage electric field. In general, electrospray consists of a syringe pump, a metal needle serving as a nozzle, a high voltage electric power supply (basically operated through direct current but also possible via alternating current) and grounded metal collector (plate or drum). The syringe pump is used to control the rate of flow of the protein solution. The rate of flow is found to be important in controlling the size of the particle. The high voltage AC power is basically operated through direct current but some reports have also used alternating current. The protein nanoparticles formed by this method have narrow polydispersity index and the loading of charged therapeutic molecules can easily be incorporated to these nanostructures with high loading efficacy. However, the loading efficiency of hydrophobic drug is a limiting task with this method. ${ }^{34}$

\section{Physicochemical parameters controlling the drug release from protein carrier}

The physicochemical properties of biopolymers are important as they can be manipulated to alter the kinetics of drug release from the carrier. These parameters include molecular weight, hydrophobicity, surface charge, the composition of the copolymer and phase transition temperature. The effect of some of these properties on drug release is summarized below.

\subsection{Molecular weight}

The drug released from biopolymers generally follows a complex mechanism which involves combination of simple diffusion of the drug through the matrix and degradation of the biopolymer matrix followed by diffusion. The degradation of the polymer shows clear dependence on the molecular weight of the polymer. Generally, during drug release, the low molecular weight polymers are found to erode fast compared to those of higher molecular weight. Park et al., provided evidence suggesting that the different degradation pattern in polymers with varying molecular weights occurs due to the differences in phase transition temperatures and crystallinity. ${ }^{35-37}$ Lai et al., investigated the effect of molecular weight of gelatin hydrogel carrier to deliver the cultivated human corneal endothelial cell (HCEC). For their studies, they prepared gelatin carrier disks, with a different IEP (5.0 and 9.0) and a molecular weight (MW) ranging from 3 to $100 \mathrm{kDa}$. The effect of IEP and MW of the raw gelatins (i.e., before irradiation) on the functionality of sterilized disks was studied by determining mechanical property, water content, dissolution degree and cytocompatibility. Irrespective of the IEP of raw gelatin, hydrogel disks prepared with high MW (100 $\mathrm{kDa}$ ) exhibited a greater tensile strength, lower water content, and slower dissolution rate than those made of low MW gelatin ( 8 and $3 \mathrm{kDa}$ ). From the investigation of cellular responses to the disks, the negatively charged gelatin (IEP $=5.0$ ) groups were found to be more cytocompatible when compared with their positively charged counterparts (IEP $=9.0)$ at the same MW (100 kDa). It is concluded that the gamma-sterilized hydrogel disks made from raw gelatins (IEP = 5.0, $\mathrm{MW}=100 \mathrm{kDa}$ ) with appropriate dissolution degree and acceptable cytocompatibility are capable of providing stable mechanical support, making these carriers promising candidates for intraocular delivery of cultivated HCEC sheets. ${ }^{38}$ 
Chen and Ofner investigated the effect of molecular weight of gelatin-methotrexate (MTX) conjugates on HL-60 leukaemia cell growth. In their study, they observed that for gelatin-MTX conjugate at low drug load (G-MTX-L), the growth inhibition of the HL-60 leukaemia cell was inversely related to the molecular weight of the gelatin-drug conjugate. They proposed that the G-MTX-L, entered cells by fluid-phase pinocytosis into an endosome, which cleaved MTX from the conjugate releasing the MTX into the cytoplasm to inhibit cell growth. The pinocytosis is hindered with higher molecular weight gelatin. ${ }^{39}$

The molecular weight of silk fibroin under the heated alkaline conditions can be inversely controlled by the duration and type degumming process. Pritchard et al., reported that the drug-release rate can be controlled by changing the molecular weight of silk. Increased degumming time resulted in a decrease in the molecular weight of the fraction. They reported that the increase in the molecular fraction resulted in the increase of silk solution viscosity, its crystallinity and phase transition temperature. Additionally, the proteolytic degradation was found to be faster with lower molecular weight fraction, indicating the release of drug from silk fibroin can be controlled by modulating their molecular weight. ${ }^{40}$ Similarly, Kim et al., investigated the effect of molecular weight of silk fibroin on the physical properties of gel. They obtained fibroin with a broad range of molecular weight $(263.1-82.7 \mathrm{kDa})$ by using alkaline hydrolysis of silk fibroin at different time (10-180 min). They reported that the change of molecular weight of silk fibroin affected their properties like swelling ratio, shear modulus, transparency as well as cell adhesion of fibroin hydrogels. This was due to the effect of molecular weight of fibroin on the construction of microscopic structure of hydrogel. ${ }^{41}$

\subsection{Shape and morphology}

Different types of protein carriers ranging from nanoparticles, hydrogels, films, etc., can have different morphology like simple sphere, rod, or sheet. The morphology and shape of biopolymers like proteins are important factors that influence the drug-delivery efficiency, which is mostly due to the change in surface to volume ratio. Cho and $\mathrm{Ku}$ prepared albumin microspheres by chemical denaturation method using terephthaloyl chloride (TP) as a crosslinking agent, and by heat denaturation method. They investigated the effect of preparation method on the shape, size distribution of albumin microspheres, loading of betamethasone sodium phosphate as drug and its in vitro release profile. The results showed that the shape of albumin microspheres prepared by chemical denaturation was irregular and rough, whereas those prepared by heat denaturation was spherical, smooth and relatively compact. The drug release from thermally denatured albumin microparticles was faster as compared to chemically cross-linked microparticles. ${ }^{42}$

Bjørge et al., studied the influence of the surface area by varying particle shape of drug carrier on their ability to release drug and cell viability. For this they developed a novel method to produce spheroidal hydrogel particles with adjustable circularity, aiming to tune drug delivery. To achieve this, droplets of hydrogel precursor were squeezed between two superamphiphobic surfaces separated with spacers with different heights, and then photo-cross linked to maintain the acquired shape after 'de-sandwiching.' Compared to the spherical-shaped hydrogel, the spheroids shaped hydrogel showed improved viability of encapsulated cells due to enhanced nutrient diffusion to the core, and led to a significantly faster drug release rate from the polymer network. This higher efficiency of the spheroid shape may be attributed to their higher surface area to volume ratio. These results were also assessed numerically, in which the drug release rate was computed for different spheroidal-like geometries. $^{43}$

In another study, Wang et al., discovered the effect of applied voltage, on the morphology of electrospun zein protein fabrics. They reported that these protein fabrics formed either flat sheets or self-rolled tubes when immersed in water on the application of different voltage. The zein fibers fabricated at relatively lower voltage were stabilized by the pre-aggregated nanoscale hydrophobic domains and exhibited restricted swelling with flat sheet shape morphology. In the presence of higher voltage, greater bending instability was induced during the electrospinning process, and the zein network structure formed self-rolling of the aligned fibers. Sessile drop measurements showed a reduction in the contact angle from $106^{\circ}$ to $39^{\circ}$ for the fibers with $50 \%$ zein prepared at raised voltage, indicating the conversion of surface properties caused by the relaxation. These electrospun fabrics prepared at higher voltage exhibited strong resistance to protein adsorption and cell attachment, and the release experiment indicated that both three-dimensional porous structures could serve as a carrier for controlled release of incorporated bioactive compounds into phosphate-buffered saline. This phenomenon opens up many potential biomedical applications for drug delivery where the carrier does not attach to any cells or proteins. ${ }^{44}$ 
Banerjee et al., investigated the uptake of polystyrene derived nanoparticles of similar size but varying in shape such as sphere-, rod- and disc-shaped particles in Caco- 2 and Caco-2/HT-29 cells. Their results indicated that rod-shaped nanoparticles yield a higher efficiency in adhering to the cells as compared to the spherical nanoparticles, as rod-shaped nanoparticles have a higher surface area and the ability to interact more efficiently with the cell surface membranes with respect to their shape. ${ }^{45}$ The rate of drug release from polymeric drug delivery systems correlates with the mechanical properties of the polymer; high-molecular-weight polymers have a low elastic modulus, producing a relatively non-deformable matrix that limits the number of pore-forming channels. Low-molecular weight polymers have a high elastic modulus, and the matrix is more deformable, causing pores to expand as a result of osmotic pressure. ${ }^{3}$

\subsection{Particle size}

The effect of particle size is found to affect the release of drug from the polymers. In general, the release depends on the surface area, where the smaller particles are found to show fast release as compared to the bigger particle size. Our group investigated the role of particle size of human serum albumin (HSA) nanoparticles loaded with curcumin. For this, the nanoparticles were prepared in the size ranging from 25-250 nm by using dithiothreitol (DTT) and sodium deoxycholate $(\mathrm{NaDC})$ as reducing and stabilizing agents. The release of curcumin from the nanoparticles showed slow release as compared to the native HSA and followed a complex biphasic pattern. The cumulative release of curcumin from HSA nanoparticles the release of curcumin was found to decrease with increase in particle size. The uptake of curcumin from these nanoparticles was followed in human lung carcinoma (A549) cells. HSA nanoparticles of $\sim 125 \mathrm{~nm}$ facilitated maximum cellular uptake of curcumin suggesting it to be the optimal size for delivering hydrophobic drugs. ${ }^{46}$

Similarly, Mladenovska et al., investigated the effect of particle size of gelatin microsphere microspheres on the release and loading efficiency of radiolabel BSA protein. These microspheres were prepared by emulsification of aqueous solution of gelatin $(10 \% \mathrm{w} / \mathrm{v})$ and radiolabelled ${ }^{131}$ I-BSA $(2.5 \%$ $\mathrm{w} / \mathrm{v}$ ) into the particles in oil and stabilized by crosslinking with glutaraldehyde-saturated toluene $\left(0.05 \mathrm{mg} / \mathrm{mg}\right.$ gelatin) at $0{ }^{\circ} \mathrm{C}$. By variations in the process parameters, time of emulsification and sorbitan monooleate concentration, particles in a range of $7.03 \pm 1.23$ to $1.19 \pm 1.96 \mu \mathrm{m}$ were prepared. They observed that the release of the BSA from the microsphere exhibited biphasic behaviour, where there was a fast-initial release followed by a slow sustained release. BSA released from gelatin microspheres with a mean diameter smaller than $4 \mu \mathrm{m}$, was found to be in the range 30 and $55 \%$ at $3 \mathrm{~h}$. However, for microsphere greater than $5 \mu \mathrm{m}$, for the same amount of BSA $(\approx 37 \%)$ the time required was $\mathrm{s} \approx 6 \mathrm{~h}$, indicating that higher size exhibited slow release. ${ }^{47}$

Similarly, Leucuta et al., studied the release of Oxprenolol from bioadhesive microsphere prepared by mixing gelatin/poly (acrylic acid) using emulsion technique in the presence of sunflower oil. By changing the concentration of glutaraldehyde, different sizes of microspheres ranging from $50-500 \mu \mathrm{m}$ were prepared. The release of the drug followed biphasic behaviour due to the release by a combination of diffusion and relaxation mechanism. The release kinetics from the microsphere was dependent on particle size and larger particle showed slow release compared to a smaller particle. For the smaller particle size, the release was dominated by simple diffusion mechanism, while the contribution from relaxation mechanism increased with particle size. ${ }^{48}$

\subsection{Glass transition temperature}

Another factor that influences polymer physicochemical properties is the glass transition temperature $\left(T_{g}\right)$ of polymers. The $\mathrm{T}_{\mathrm{g}}$ of the polymer is dictated by molecular arrangement in the polymer and its molecular weight. The change in $T_{g}$ can be brought out by the presence of additives and it can be modulated to improve dissolution, bioavailability, processing and handling qualities of the polymeric material. Generally, below the $\mathrm{T}_{\mathrm{g}}$, the polymer is in a glassy state; i.e., it has limited mobility and low diffusion rates. Above the $T_{g}$, the polymer is in a visco-elastic state, which facilitates diffusion of water and drug molecules throughout the matrix. For an effective design of degradable polymers in drug delivery applications, a balance between amorphous and crystalline states is necessary, as these parameters have direct effects on the degree of mechanical toughness and rate of drug release. During the blending of biopolymers with plasticizers, the bounded water molecules are found to influence the glass transition due to its plasticization in the protein structure, as reported in wheat and soy proteins. ${ }^{49,50}$ The effect of additive can result in an increase or decrease on the $\mathrm{T}_{\mathrm{g}}$ of the composite polymer. Treesuppharat et al., 
investigated the influence of bacterial cellulose on the $\mathrm{T}_{\mathrm{g}}$ of the gelatin hydrogel and found it to increase to 60 ${ }^{\circ} \mathrm{C}$ in presence of bacterial cellulose. ${ }^{51}$ Pritchard et al., reported that decrease in $T_{g}$ of silk fibroin protein resulted in increases in the drug release rate. ${ }^{40}$

\subsection{Porosity}

In nanoparticles, the surface area to volume ratio is higher compared to bulk. However, the presence of pore can also increase the total surface area and solvent permeability. Thus, altering the porosity of a material can be used to control the loading and release of drug. These parameters can also be altered by pore size and its interconnectivity of the material. Increasing the porosity can enhance degradation and increase drug release in a polymer matrix, largely by the surface area to volume ratio. Schnieders et al., investigated the influence of porosity on the release kinetics of vancomycin from calcium phosphate cement composites. They changed the porosity by changing the ratio of cement powder to the liquid (water). They observed that higher porosity of cement composite resulted in higher drug release from the composite. ${ }^{52}$ In order to achieve rapid drug delivery with a low percentage of pores, Itokazu et al., developed an antibiotic-loaded hydroxyapatite ceramic scaffold with uniformly accessible pores, interlinked pore system, and enlarged macropores. ${ }^{53}$

\section{Surface modification of proteins}

The efficacy of protein nanoparticles can be increased by a modification which generally plays an important role in improving nanoparticle's targeting and stability, leading to their high efficiency as drug carriers. Polyethylene glycol is used to increase the stability of the nanoparticles as it reduces reticuloendothelial system (RES) uptake and prolonges circulation time. The PEG group creates a hydrophilic barrier that results in blocking electrostatic and hydrophobic interactions responsible for opsonization. Similarly conjugating protein nanoparticles with low molecular weight molecules like hyaluronic acid, glycyrrhizic acid, sugars, etc., have found to increase the efficiency of these nanocarriers. ${ }^{54-56}$ Some of the ligands that are reported to be conjugated with proteins are given in Table 1.

\section{Advantages and disadvantages of protein nanoparticles}

Proteins being naturally occurring biopolymers have the advantages of being mucoadhesive, non-toxic, biocompatible, biodegradable and therefore can be readily used for oral or bulk matrix delivery. These materials on degradation forms end products that are not toxic and can be readily eliminated out of the body. With these advantages, there are certain challenges that limit the translation of the material to commercial utility. These biomaterials have been reported to have fast drug release due to faster biodegradability. For improving the stability of the protein nanoparticles, they have to be cross-linked. The presence of crosslinked protein nanoparticle can induce immune/inflammatory response. For industrial scalability, it is difficult to optimize an economical method to obtain clinical grade material. This can lead to batch to batch variation. Due to a natural origin, they can be contaminated with pathogen from other species. All these factors can lead to regulatory issues for commercial approval. ${ }^{57}$ The advantages and disadvantages of the protein discussed in the present article are summarized in Table 2.

\section{Challenges in developing protein-based carriers for commercial use}

Recent academic research on utilization of proteins as smart materials for drug delivery supports the utility of these biopolymers as cost-effective, biocompatible and

Table 1. Surface modification of proteins with different ligands to enhance the drug delivery efficiency.

\begin{tabular}{lll}
\hline Ligand & \multicolumn{1}{c}{ Protein } & \multicolumn{1}{c}{ System } \\
\hline Folate & Albumin & Human prostate cancer cells (PC3 cells) \\
Antibody against PSMA & Apoferritin & LNCaP cells induced tumour in athymic mice model \\
Arginylglycylasparticacid (RGD) peptides & Albumin & C26-tumour-bearing Balb/C mice \\
Biotinylated HER2 antibody & HSA and gelatin & HER2 over expressing cells \\
Apo-transferrin & Albumin & MCF-7 and A549 cells \\
Bisphosphonate & BSA & Human C2C12 cells and rat bone marrow stromal cells \\
Apolipoprotein E & Albumin & Crosses blood brain barrier in ICR mice \\
\hline
\end{tabular}




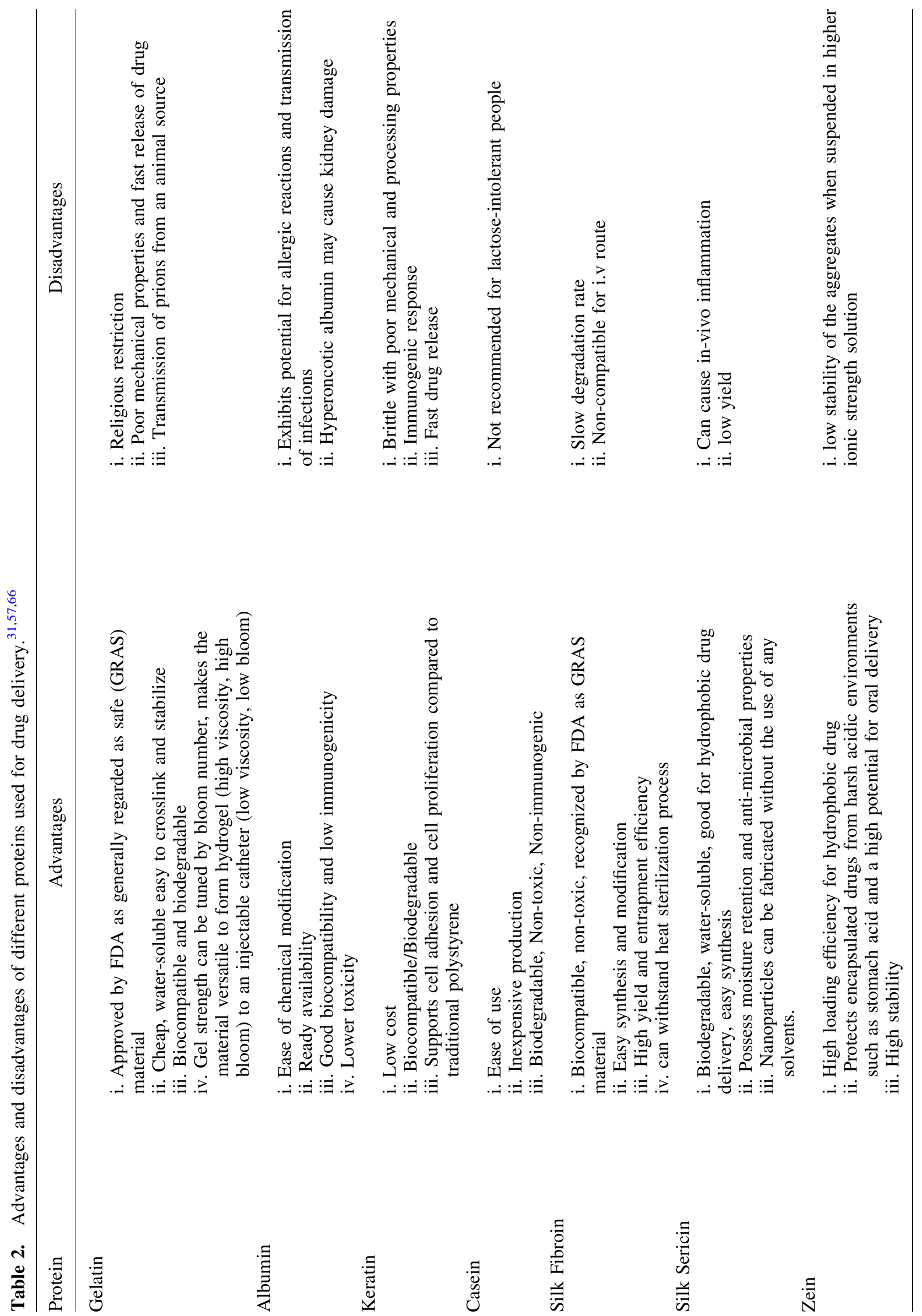


biodegradable carriers. Reports in the literature highlight that naturally occurring proteins, as well as engineered proteins, have potential applications in delivering anti-cancer drugs, peptide hormones, growth factors and even in the delivery of genetic material like DNA and RNA. However, there are technical bottlenecks for translating these materials from the research lab to the clinic. Some of the intrinsic concerns using proteins are structural changes during fabrication that may result in loss of activity of the native protein, presence of endotoxin or transmission of prions, low yield of some protein-based nanoparticles and rapid degradation. ${ }^{31} \mathrm{~A}$ lot of effort is being devoted to overcoming these challenges. Low yield in case of protein nanoparticles can be countered by using the recombinant protein approach along with further research on improving its downstream processing. The presence of endotoxin in recombinant proteins can be prevented by using yeast or plants as an expression system. Blending of protein with other biocompatible polymers can be used to tailor physicochemical properties of protein-based nanoparticles and address the problems of rapid drug release. ${ }^{31}$ Along with these, there are challenges in developing these materials for commercial development. The main hurdles come in scaling up the synthesis protocol for larger commercial production. Generally, during the preparation of nanoparticles, a subtle variation in the manufacturing procedure may result in significant changes in their physicochemical properties. This may have a drastic effect on the efficacy of the nanocarrier. Further, for clinical use the product must be sterilized. Gamma irradiation or autoclaving of the biomaterial may damage the therapeutics along with the carrier. Thus sterilization can be done by filtering the material before lyophilisation. However, the size of the carrier along with the drug should be less than $0.22 \mu \mathrm{m}$. These limitations can be overcome by avoiding multistep processes for fabrication which will also result in the economical suitability of the process. Additionally, regulatory aspect is also an uphill procedure in commercializing the product. Regulatory agencies like FDA, EMA, and CDSCO require delivery system to complete the preclinical and clinical evaluation process even if the active pharmaceutical ingredient (API) has been used regularly in the clinic. ${ }^{58}$ Even with these restrictions, certain protein-based products such as Abraxane $^{\circledR}$, ABI-008 (Paclitaxel albumin-bound nanoparticles) for metastatic breast cancer, Onto ${ }^{\circledR}$, (Denileukin Diftitox, FDA approved engineered fusion protein treat non-Hodgkin's Peripheral T-cell Lympomas) and Gelafundin, Gelafusal as plasma expanders for intravenous infusion has been approved by regulatory agency and are being commercially successful. ${ }^{31,59}$

\section{Conclusions}

Protein-based polymers are becoming prevalent in their application in medicinal and pharmaceutical fields. Besides having added advantage of being biocompatible and biodegradable, there are some challenges to be addressed for making the practical and commercial protein-based carrier. Some of these are controlling their biodegradation, mechanical strength, particle distribution and stability. This review summarized on the structural properties of certain proteins, their merits and demerits and the factors that decide the drug-releasing ability of different forms to achieve better application. As research continues, more fabrication methods will be developed to address the challenges in developing these materials for commercial purpose.

\section{Acknowledgements}

The authors acknowledge Drs. V. K. Jain (Director, DAECBS), K. I. Priyadarsini (Head, Chemistry Division, BARC), and Dr. P. A. Hassan, Chemistry Division BARC, for their support and encouragement.

\section{Reference}

1. Öztürk-Atar K, Eroğlu H and Calis S 2018 Novel advances in targeted drug delivery J. Drug Target 26 633

2. Jahangirian H, GhasemianL E, Webster T J, RafieeMoghaddam R and Abdollahi Y 2017 A review of drug delivery systems based on nanotechnology and green chemistry: green nanomedicine Int. J. Nanomed. 12 2957

3. Kamaly N, Yameen B, Wu J and Farokhzad O C 2016 Degradable controlled release polymers and polymeric nanoparticles: Mechanisms of controlling drug release Chem. Rev. 1162602

4. Lohcharoenkal W, Wang L, Chen Y C and Rojanasakul Y 2014 Protein nanoparticles as drug delivery carriers for cancer therapy Biomed. Res. Int. 2014180549

5. Spicer C D, Jumeaux C, Gupta B and Stevens M M 2018 Peptide and protein nanoparticle conjugates: versatile platforms for biomedical applications Chem. Soc. Rev. 473574

6. Verma D, Gulati N, Kaul S, Mukherjee S and Nagaich U 2018 Protein based nanostructures for drug delivery J. Pharm. 2018 Article ID 9285854

7. Jao D, Xue Y, Medina J and Hu X Protein based drug delivery materials Materials 10517

8. Tarhinia M, Greige-Gerges H and Elaissaria A 2017 Protein-based nanoparticles: From preparation to 
encapsulation of active molecules Int. J. Pharm. 522 172

9. https://en.wikipedia.org/wiki/Protein

10. Foxx M and Zilberman M 2015 Drug delivery from gelatin-based systems Expert Opin. Drug Deliv. 12 1547

11. Santoro M, Tatara A M and Mikosa A G 2014 Gelatin carriers for drug and cell delivery in tissue engineering J. Control. Release 190210

12. Young S, Wong M, Tabata Y and Mikos A G 2005 Gelatin as a delivery vehicle for the controlled release of bioactive molecules. J. Control. Release 109256

13. Sakai A, Murayama Y, Fujiwara K, Fujisawa T, Sasaki S, Kidoaki S and Yanagisawa M 2018 Increasing elasticity through changes in the secondary structure of gelatin by gelation in a microsized lipid space $A C S$ Cent. Sci. 4477

14. Larsen M T, Kuhlmann M, Hvam M L and Howard K A 2016 Albumin-based drug delivery: harnessing nature to cure disease Mol. Cell. Therap. 43

15. Liu F, Mu J and Xing B 2015 Recent advances on the development of pharmacotherapeutic agents on the basis of human serum albumin Curr. Pharm. Des. 21 1866

16. Merlot A M, Kalinowski D S and Richardson D R 2014 Unraveling the mysteries of serum albumin-more than just a serum protein Front Physiol. 12299

17. Dennis M S, Zhang M, Meng Y G, Kadkhodayan M, Kirchhofer D, Combs D and Damico L A 2002 Albumin binding as a general strategy for improving the pharmacokinetics of proteins J. Biol. Chem. 27735035

18. Sleep D 2015 Albumin and its application in drug delivery Expert Opin. Drug Deliv. 12793

19. Shavandi A, Silva T H, Bekhit A A and Bekhit A E A 2017 Keratin: dissolution, extraction and biomedical application Biomater. Sci. 51699

20. Mogosanu G D, Grumezescu A M and Chifiriuc M C 2014 Keratin-based biomaterials for biomedical applications, Keratin-based biomaterials for biomedical applications Curr. Drug Targets 15518

21. Rouse J G and Van D M E 2010 A review of keratinbased biomaterials for biomedical applications Materials 3999

22. Kure J M, Pierlot A P, Russell I M and Shanks R A 1997 The glass transition of wool: An improved determination using DSC Text. Res. J. 6718

23. Głąb T K and Boratyński J 2017 Potential of casein as a carrier for biologically active agents Top. Curr. Chem. 37571

24. Elzoghby A O, El-Fotoh W S and Elgindy N A 2011 Casein-based formulations as promising controlled release drug delivery systems J. Control. Release 153206

25. Horne D S 2002 Casein structure, self-assembly and gelation Curr. Opin. Colloid. Interface Sci. 7456

26. Numata K and Kaplan D L 2010 Silk-based delivery systems of bioactive molecules Drug Deliv. Rev. 62 1497

27. Lamboni L, Gauthier M, Yang G and Wang Q 2015 Silk sericin: A versatile material for tissue engineering and drug delivery Biotechnol. Adv. 331855

28. Huang W, Ling S, Li C, Omenetto F G and Kaplan D L 2018 Silkworm silk-based materials and devices generated using bio-nanotechnology Chem. Soc. Rev. 476486

29. Corradini E, Curti P S, Meniqueti A B, Martins A F, Rubira A F and Muniz E C 2014 Recent advances in food-packing, pharmaceutical and biomedical applications of zein and zein-based materials Int. J. Mol. Sci. 1522438

30. Demir M, Ramos-Rivera L, Silva R, Nazhat S N and Boccaccini A R 2017 Zein-based composites in biomedical applications J. Biomed. Mater. Res. A 105 1656

31. Jain A, Singh S K, Arya S K, Kundu S C and Kapoor S 2018 Protein nanoparticles: promising platforms for drug delivery applications ACS Biomater. Sci. Eng. 12 3939

32. Wang G and Uludag H 2008 Recent developments in nanoparticle-based drug delivery and targeting systems with emphasis on protein-based nanoparticles Expert Opin. Drug Deliv. 5499

33. Kim K J and Byun Y 1999 Preparation and characterizations of self-assembled PEGylated gelatin nanoparticles Biotechnol. Bioprocess. Eng. 4210

34. Pawar A, Thakkar S and Misra M 2018 A bird's eye view of nanoparticles prepared by electrospraying: Advancements in drug delivery field J. Control. Release 286179

35. Park T G 1995 Degradation of poly (lactic-co-glcocic acid) microspheres: effect of copolymer composition Biomaterials 161123

36. Park T G 1994 Degradation of poly(d,1-lactic acid) microspheres: effect of molecular weight J. Control. Release 30161

37. Freiberg S and Zhu X X 2004 Polymer microspheres for controlled drug release Int. J. Pharm. 2821

38. Lai J Y, Lu P L, Chen K H, Tabatal Y and Hsiue G H 2006 Effect of charge and molecular weight on the functionality of gelatin carriers for corneal endothelial cell therapy Biomacromolecules 71836

39. Chen C S and Ofner C M 2009 The effect of molecular weight, drug load, and charge of gelatin-MTX conjugates on growth inhibition of HL-60 leukemia Cells Pharm. Res. 26338

40. Pritchard E M, Hu X, Finley V, Kuo C K and Kaplan D L 2013 Effect of silk protein processing on drug delivery from silk films Macromol. Biosci. 13311

41. Kim H H, Song D W, Kim M J, Ryu S J, Um I C, Ki C S and Par Y H 2016 Effect of silk fibroin molecular weight on physical property of silk hydrogel Polymer 9026

42. Kwon G S S 1991 Albumin-heparin microspheres for drug delivery Thesis submitted to (University of Utah, Utah) p. 241

43. Bjørge I M, Costa A M S, Silva A S, Vidal J P O, Nóbrega J M and Mano J F 2018 Tuneable spheroidal hydrogel particles for cell and drug encapsulation Soft Matter. 145622

44. Wang Y, Yang J and Chen L 2015 Convenient fabrication of electrospun prolamin protein delivery system with three-dimensional shape ability and resistance to fouling ACS Appl. Mater. Interfaces 713422

45. Banerjee A, Qi J, Gogoi R, Wong J and Mitragotri S 2016 Role of nanoparticle size, shape and surface 
chemistry in oral drug delivery $J$. Control. Release 238 176

46. Das R P, Gandhi V V, Singh B G, Kunwar A, Kumar N and Priydarsini K I 2019 Preparation of albumin nanoparticles: Optimum size for cellular uptake of entrapped drug (Curcumin) Colloids. Surf. A $\mathbf{5 6 7} 86$

47. Mladenovska K, Kumbaradzi E F, Dodov G M, Makraduli L and Goracinova K 2002 Biodegradation and drug release studies of BSA loaded gelatin microspheres Int. J. Pharm. 242247

48. Leucuta S E, Ponchel G and Duchêne D 1997 Oxprenolol release from bioadhesivegelatin/poly(acrylic acid) microspheres J. Microencapsul. 14511

49. Noel T R, Parker R, Ring S G and Tatham A S 1995 The glass-transition behaviour of wheat gluten proteins Int. J. Biol. Macromol. 1781

50. Mizuno A, Mitsuiki M, Motoki M, Ebisawa K and Suzuki E 2000 Relationship between the glass transition of soy protein and molecular structure J. Agric. Food. Chem. 483292

51. Treesuppharat W, Rojanapanthu P, Siangsanoh C, Manuspiya H and Ummartyotin S 2017 Synthesis and characterization of bacterial cellulose and gelatin-based hydrogel composites for drug-delivery systems Biotechnol. Rep. 1584

52. Schnieders J, Gbureck U, Vorndran E, Schossig M and Kissel T 2011 The effect of porosity on drug release kinetics from vancomycin microsphere/calcium phosphate cement composites J. Biomed. Mater. Res. B Appl. Biomater. 99391

53. Itokazu M, Yang W, Aoki T, Ohara A and Kato N 1998 Synthesis of antibiotic-loaded interporous hydroxyapatite blocks by vacuum method and in vitro drug release testing Biomaterials 19817

54. Chen Z, Chen J, Wu L, Li W, Chen J, Cheng H, Pan J and Cai B 2013 Hyaluronicacid-coated bovine serum albumin nanoparticles loaded with brucine as selective nanovectors for intra-articular injection Int. J. Nanomed. 83843

55. Zu Y, Meng L, Zhao X, Ge Y, Yu X, Zhang Y and Deng Y 2013 Preparation of 10-hydroxycamptothecinloaded glycyrrhizic acid-conjugated bovine serumalbumin nanoparticles for hepatocellular carcinoma-targeted drug delivery Int. J. Nanomed. 81207

56. Davis B G and Robinson M A 2002 Drug delivery systems based on sugar-macromolecule conjugates Curr. Opin. Drug Discov. Dev. 5279

57. Martins A, Ferreira H, Reis R L and Neves N M 2016 Delivery systems made of natural-origin polymers for tissue engineering and regenerative medicine applications in biomaterials from nature for advanced devices and therapies R L Reis and N M Neves (Eds.) (Hoboken: Wiley) p. 583

58. Ragelle H, Danhier F, Préat V, Langer R and Anderson D G 2017 Nanoparticle-based drug delivery systems: a commercial and regulatory outlook as the field matures Expert Opin. Drug Deliv. 14851

59. Zhao D, Zhao X, Zu Y, Li J, Zhang Y, Jiang R and Zhang Z 2010 Preparation, characterization, and in vitro targeted delivery of folate-decorated paclitaxelloaded bovine serum albumin nanoparticles Int. J. Nanomed. 5669

60. Dostalova S, Polanska H, Svobodova M, Balvan J, Krystofova O, Haddad Y, Krizkova S, Masarik M, Eckschlager T, Stiborova M, Heger Z and Adam V 2018 Prostate-specific membrane antigen-targeted sitedirected antibody-conjugated apoferritin nanovehicle favorably influences in vivo side effects of doxorubicin Sci. Rep. 88867

61. Temming K, Meyer D L, Zabinski R, Dijkers E C, Poelstra K, Molema G and Kok R J 2006 Evaluation of RGD-targeted albumin carriers for specific delivery of auristatin E to tumor blood vessels Bioconjug. Chem. 171385

62. Wartlick H, Michaelis K, Balthasar S, Strebhardt K, Kreuter J and Langer K 2004 Highly specific HER2mediated cellular uptake of antibody-modified nanoparticles in tumour cells J. Drug. Target. 12461

63. Choi J S and Meghani N 2016 Impact of surface modification in BSA nanoparticles for uptake in cancer cells Colloids Surf. B Biointerfaces 145653

64. Wang G, Kucharski C, Lin X and Uludağ H 2010 Bisphosphonate-coated BSA nanoparticles lack bone targeting after systemic administration J. Drug Target. 18611

65. Michaelis K, Hoffmann M M, Dreis S, Herbert E, Alyautdin R N, Michaelis M, Kreuter J and Langer K 2006 Covalent linkage of apolipoprotein e to albumin nanoparticles strongly enhances drug transport into the brain J. Pharmacol. Exp. Ther. 3171246

66. Tapia-Hernández J A, Rodríguez-Felix F, JuárezOnofre J E, Ruiz-Cruz S, Robles-García M A, Borboa-Flores J, Wong-Corral F J, Cinco-Moroyoqui F J, Castro-Enríquez D D and Del-Toro-Sánchez C L 2018 Zein-polysaccharide nanoparticles as matrices for antioxidant compounds: A strategy for prevention of chronic degenerative diseases Food Res. Int. 111 451 